\title{
COUNTEREXAMPLES ON THE FUSION OF INVOLUTIONS IN FINITE GROUPS
}

\author{
STEPHEN DORO
}

\begin{abstract}
ABSTRACr. Some examples are given of simple groups $G$ containing a conjugacy class of involutions $\mathcal{C}$ such that if $S$ is a Sylow 2-subgroup of $G$,

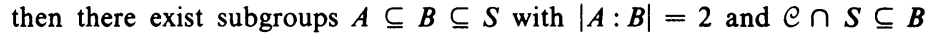
$-A$.
\end{abstract}

Three conjectures arise naturally in the study of the fusion of involutions in finite groups.

Conjecture 1. Let $G$ be a finite group. Let $C$ be a union of conjugacy classes of involutions and let $S \in \operatorname{Syl}_{2}(G)$. For $H \subseteq G$, define $K_{H}=\operatorname{gp}\langle a b| a, b$

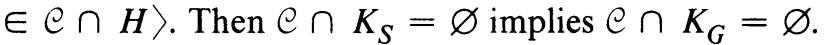

Conjecture 2. Let $G$ be a finite group and let $\mathcal{C}$ and $\mathscr{D}$ each be unions of conjugacy classes of involutions. Let $S \in \operatorname{Syl}_{2}(G)$. Then $\mathcal{\cap} \cap\langle\mathcal{D} \cap S\rangle=\varnothing$ implies $e \cap\langle\mathscr{D}\rangle=\varnothing$.

Conjecture 3. Let $G$ be a finite group and let $C$ and $\mathscr{D}$ each be unions of conjugacy classes of involutions. Let $S \in \operatorname{Syl}_{2}(G)$. For $H \subseteq G$, let $L_{H}$ $=\langle a, b c \mid a \in \mathscr{D} \cap H, b, c \in \mathcal{C} \cap H\rangle$. Then $L_{S} \cap \mathcal{C}=\varnothing$ implies $\langle\mathscr{D}\rangle \cap \mathcal{C}$ $=\varnothing$.

In the special case when $K_{S}=1$, Conjecture 1 is equivalent to Glauberman's $Z^{*}$ theorem; in the case when $\left|S: K_{S}\right|=2$, Conjecture 1 is equivalent to the Thompson transfer lemma. Conjecture 1 is an attempt to generalize these results.

Conjecture 3 is not only an immediate consequence of Conjecture 2; it is also a special case of Conjecture 1. Namely, assuming Conjecture 1 and the hypotheses of Conjecture 3 , let $G_{0}=\langle z\rangle \times G$, where $z^{2}=1$, let $C_{0}=\mathcal{C}$ $\cup z \mathscr{D}$ and apply Conjecture 1 to $G_{0}$ and $\bigodot_{0}$ to derive the conclusion of Conjecture 3 . The purpose of this note is to point out an infinite series of counterexamples to Conjecture 3 and hence to Conjectures 1 and 2 .

The groups to be discussed are the simple groups $\operatorname{PSL}_{8}(q)$ where $q$ is a prime power with $q \equiv 1 \bmod 16$. By Dirichlet's theorem, there are infinitely many suitable $q$, of which the smallest is $q=17$. The example is based upon consideration of the natural representation of $\mathrm{SL}_{8}(q)$ on an 8-dimensional vector space over $\operatorname{GF}(q)$. Throughout the discussion, therefore, let $G$

Received by the editors December 4, 1975.

AMS (MOS) subject classifications (1970). Primary 20D05, 20D20; Secondary 20G40.

Key words and phrases. Involutions, fusion, Sylow 2-subgroups, $Z^{*}$ theorem.

(c) American Mathematical Society 1976 
$=\mathrm{SL}_{8}(q), \mathbf{Z}=\mathbf{Z}\left(\mathrm{SL}_{8}(q)\right), \bar{G}=\mathrm{PSL}_{8}(q)=G / \mathbf{Z}$ with the usual bar convention for subgroups. Let $V$ be an 8-dimensional vector space over $\operatorname{GF}(q)$ which faithfully represents $G$ and fix an $S \in \operatorname{Syl}_{2}(G)$.

The first step is to show $S$ is monomial on $V$. Let $F_{1}, F_{2}, \ldots, F_{8}$ be a collection of 1-dimensional subspaces of $V$ which span $V$ and let $G_{0}$ be the subgroup of all unimodular transformations which permute the subspaces $\left\{F_{i} \mid 1 \leqslant i \leqslant 8\right\}$ and such that the induced permutations preserve the systems of imprimitivity $\left\{\left\{F_{1}, \ldots, F_{4}\right\},\left\{F_{5}, \ldots, F_{8}\right\}\right\}$ and $\left\{\left\{F_{1}, F_{2}\right\},\left\{F_{3}, F_{4}\right\},\left\{F_{5}, F_{6}\right\},\left\{F_{7}\right.\right.$, $\left.\left.F_{8}\right\}\right\}$. If $x \in G_{0}$, let $x^{\pi}$ be the corresponding permutation of the $\left\{F_{i} \mid 1 \leqslant i\right.$ $\leqslant 8\}$ and let $D_{0}$ be the diagonal subgroup of group elements fixing all $F_{i}$. Then $G_{0} / D_{0}$ is isomorphic to $Z_{2} \sim\left(Z_{2} \times Z_{2}\right)$, a Sylow 2-subgroup of $A_{8}$. Fix an $S_{0} \in \operatorname{Syl}_{2}\left(G_{0}\right)$ and let $D=S_{0} \cap D_{0}$, so that $S_{0} / D \cong Z_{2} \sim\left(Z_{2} \times Z_{2}\right)$. Since $S_{0}$ is transitive on $\left\{F_{i} \mid 1 \leqslant i \leqslant 8\right\}, \mathbf{C}_{\mathrm{GL}_{8}(q)}\left(S_{0}\right)=\mathbf{Z}\left(\mathrm{GL}_{8}(q)\right)$ so that the representation of $S_{0}$ on $V$ is absolutely irreducible. We may choose $S$ $\in \operatorname{Syl}_{2}(G)$ with $S \supseteq S_{0}$ and then the representation of $S$ on $V$ is absolutely irreducible. Since $(q, 2)=1$ and GF $(q)$ contain a primitive 4-root of 1 , the representation of $S$ on $V$ is monomial and this forces $S=S_{0}[1,(14.4)]$. This equality could also be derived purely from group order considerations.

Let $\lambda$ be a primitive 16-root of 1 in $\operatorname{GF}(q)$, fix nonzero basis elements $f_{i} \in F_{i}$ for $1 \leqslant i \leqslant 8$ and in this basis define elements of $G$;

$$
c=\operatorname{diag}(\lambda, \lambda, \lambda, \lambda, \lambda, \lambda, \lambda,-\lambda), \quad d=\operatorname{diag}(1,1,1,1,1,1,-1,-1) .
$$

Let $\mathcal{C}=\operatorname{ccl}(c), \mathscr{D}=\operatorname{ccl}(d)$. Let $T$ be the stabilizer in $S$ of the sets $\left\{F_{1}, \ldots, F_{4}\right\}$ and $\left\{F_{5}, \ldots, F_{8}\right\}$.

The second step is to observe that if $h \in \mathcal{D} \cap S$ then $h \in T$. For otherwise, $h^{\pi}$ consists of four transpositions. $h$ fixes four corresponding 2-dimensional subspaces; but then, since $h$ has 6 eigenvalues $1, h$ is the identity on one space $\left\langle F_{u}, F_{v}\right\rangle, 1 \leqslant u<v \leqslant 8$, contradicting the fact that $h^{\pi}$ is a transposition on $\left\{F_{u}, F_{v}\right\}$. Similarly, if $g \in e \cap S$, then $g \in T$.

Now define a homomorphism $\delta: T Z \rightarrow Z_{2^{a}}$ by $\delta(x)=\left.\operatorname{det} x\right|_{\left\langle F_{1}, \ldots, F_{4}\right\rangle}$. If $z \in \mathbf{Z}$ then $\delta(z)= \pm 1$. Also $\delta(h)= \pm 1$ for $h \in \mathcal{D} \cap S(\subseteq T)$. However, if

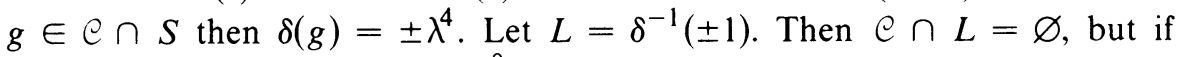
$g, h \in \mathcal{C} \cap S$ then $\delta(g h)= \pm \lambda^{8}= \pm 1$ so $g h \in L$. In $\bar{G}$, $\mathcal{C}$ and $\mathscr{D}$ become conjugacy classes of involutions and if $L_{S}$ is defined as in Conjecture 3 then $\bar{L} \supseteq L_{S}$. $\cap \cap \bar{L}=\varnothing$ so $\bigodot \cap L_{S}=\varnothing$. However $\langle\mathcal{D}\rangle=\bar{G}$, by the simplicity of $\operatorname{PSL}_{8}(q)$. This example disproves all the conjectures. In particular, the class

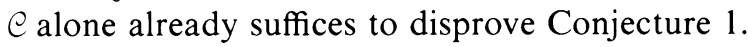

\section{REFERENCES}

1. Walter Feit, Characters of finite groups, Benjamin, New York, 1967. MR 36 \#2715.

Department of Mathematics, Yale University, New Haven, Connecticut 06520

Current address: Department of Mathematics, Michigan State University, East Lansing, Michigan 48823 